\title{
Voltage Sensor Based on a Plastic Optical Fiber with a Rectangular Hole
}

\author{
Hyejin Seo, Jonathan Prabowo, and Jaehee Park ${ }^{*}$ \\ Department of Electronic Engineering, Keimyung University, \\ 1095, Dalgubeol-daero, Dalseo-gu, Daegu 42601, Korea \\ (Received January 7, 2021; accepted March 9, 2021)
}

Keywords: plastic optical fiber sensor, in-fiber hole, voltage sensing, polymer-dispersed liquid crystal

In this paper, we present an $\mathrm{AC}$ voltage sensor based on a plastic optical fiber (POF) with a rectangular hole filled with a polymer-dispersed liquid crystal (PDLC) film. The dimensions of the rectangular hole and PDLC film used in the experiment were approximately $2 \times 0.65 \mathrm{~mm}^{2}$ and $20 \times 0.6 \times 0.2 \mathrm{~mm}^{3}$, respectively. The root-mean-square (RMS) amplitude of AC voltage at $60 \mathrm{~Hz}$ from 0 to $80 \mathrm{~V}$ was measured using this sensor. The transmittance of this sensor was a nonlinear function of the applied voltage. In the approximate linear region from 8 to $23 \mathrm{~V}$, the sensitivity of this sensor was approximately $0.017 \mathrm{~V}^{-1}$. The results show that the proposed voltage sensor can be used to measure the RMS amplitude of AC voltage.

\section{Introduction}

The plastic optical fiber (POF) has attracted considerable attention for sensing applications owing to its low cost and high repeatability of optical properties. Thus, various sensors based on POF have been developed for measuring pressure, temperature, gripping force, and refractive index. ${ }^{(1-4)}$ Voltage-sensing technologies based on POF possess some advantages over conventional voltage sensors, such as immunity to electromagnetic interference, low weight, small size, intrinsically safe mode of operation, and suitability for remote sensing; they also possess some advantages over glass fiber voltage-sensing technologies, such as ease of connection and good mechanical reliability owing to their high numerical aperture and huge core diameter. ${ }^{(5)}$ Hence, they have attracted interest for improving the performance of electrical power networks and for measuring the amplitude of AC voltage. ${ }^{(6,7)}$

Several voltage-sensing technologies based on the electro-optical Pockels effect have already been proposed ${ }^{(8,9)}$ with controllable sensitivity and measurement range. However, they provide low sensitivity. Phase-modulated glass fiber sensing technologies for voltage measurements have been proposed using the Mach-Zehnder interferometer, ${ }^{(10)}$ Michelson interferometer, ${ }^{(11)}$ Fabry-Perot interferometer, ${ }^{(12)}$ and Bragg grating. ${ }^{(13)}$ While they offer high sensitivity, they experience undesirable external perturbations and cannot provide accurate measurements in the field. Thus, the voltage-sensing technologies based on POF have also been developed because they are unaffected by ambient noise although they are less sensitive due to the

*Corresponding author: e-mail: jpark@kmu.ac.kr https://doi.org/10.18494/SAM.2021.3259 
intensity modulation. ${ }^{(14)}$ A POF voltage sensor using electro-optic quart waveplates ${ }^{(15)}$ has been investigated. It has a simple structure but low sensitivity. Another POF sensor based on polymer-dispersed liquid crystal (PDLC) films ${ }^{(16)}$ has been reported. It has sufficient sensitivity to measure AC voltage. However, it is not suitable for use in the field. Accordingly, in this paper, we present a POF-based voltage sensor with sufficient sensitivity to measure AC voltage in the field and with strong resistance to ambient noise. This sensor consists of a POF with a rectangular hole ${ }^{(17)}$ filled with a PDLC film.

\section{Background}

The PDLC film consists of micrometer-sized liquid crystal (LC) droplets in a polymer matrix. The film appears semi-transparent because of the light scattering generated by the mismatch between the refractive indices of the LC droplets and the polymer matrix. When an external alternating electric field sufficient to match the ordinary refractive index of the LC droplets to the refractive index of the polymer is applied, the film changes to a hightransmittance 'on-state' (Fig. 1). At any applied voltage V, the incident light beam is partially scattered and partially transmitted. The transmittance of the PDLC film may be expressed using Beer-Lambert's Law as follows:

$$
T(V)=I(V) / I_{0}=\exp [-(\aleph \sigma(V) d)]
$$

where $I(V)$ is the light intensity received at the photodetector placed behind the PDLC, $I_{0}$ is the incident light intensity, $\boldsymbol{K}$ is the droplet concentration, $d$ is the PDLC thickness, and $\sigma(V)$ is the average scattering cross-section depending on the applied voltage. ${ }^{(16)}$ The transmittance of the PDLC film is a nonlinear function of the applied voltage, film thickness, and droplet concentration. If a PDLC film with different characteristics is used, a different transmittance will be obtained. ${ }^{(18,19)}$ Furthermore, high-amplitude or low-amplitude AC voltages can be measured if the POF voltage sensors are fabricated using the PDLC films with different characteristics. ${ }^{(20)}$ Accordingly, the measurement voltage range depends on the characteristics of the PDLC film. The applied voltage can be measured using the transmittance of the PDLC film as expressed in Eq. (1).
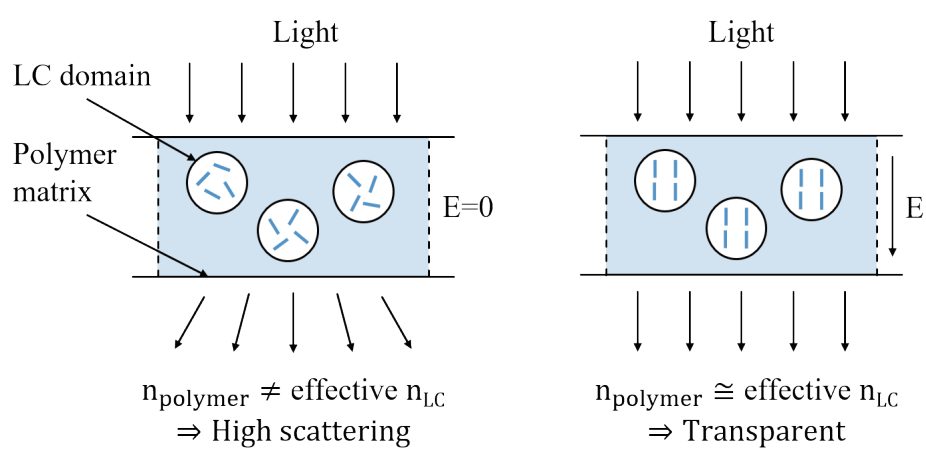

Fig. 1. (Color online) Operation principle of PDLC film. ${ }^{(18)}$ 


\section{Experiment and Results}

The POF voltage sensor with a rectangular hole was fabricated according to the procedure shown in Fig. 2. First, a rectangular hole was formed in the POF using an inexpensive drilling machine (SMC HD-280) and a micro-drill bit (NEO Technical System). ${ }^{(21)}$ Subsequently, silver paste was applied on both sides of a small-sized PDLC film for preparing electrodes. Then, two copper meshes were bonded on the silver paste area on both sides of the PDLC film using epoxy. Finally, the PDLC film with two electrodes was inserted perpendicular to the light transmission axis into the rectangular hole. The POF (Toray, PGR-FB1500) used to measure the root-meansquare (RMS) amplitude of the AC applied voltage had a core diameter of $1.48 \mathrm{~mm}$ and core and cladding indices of 1.49 and 1.41, respectively. A PDLC film (Smart Film PLDC Group Co.) with an LC-monomer mixture and a thickness of $20 \mu \mathrm{m}$ between two indium tin oxide (ITO) films was used. The light transmittance of this PDLC film at $635 \mathrm{~nm}$ wavelength was $1 \%$ (off state) $-83 \%$ (on state). The rectangular hole in the POF had the dimensions of $2 \times 0.65 \mathrm{~mm}^{2}$ and the PDLC film had the dimensions of $20 \times 0.6 \times 0.2 \mathrm{~mm}^{3}$.

The transmittance of the POF voltage sensor with the rectangular hole filled with a PDLC film was obtained with the experimental setup (Fig. 3). The experimental setup consisted of a
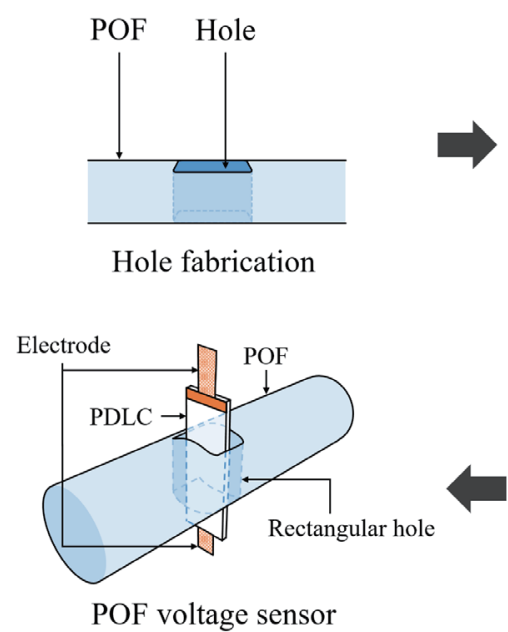

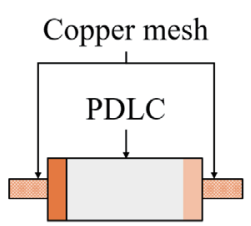

Making electrodes

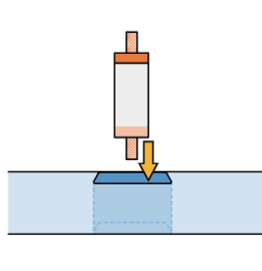

Insertion

Fig. 2. (Color online) Sensor fabrication procedures.

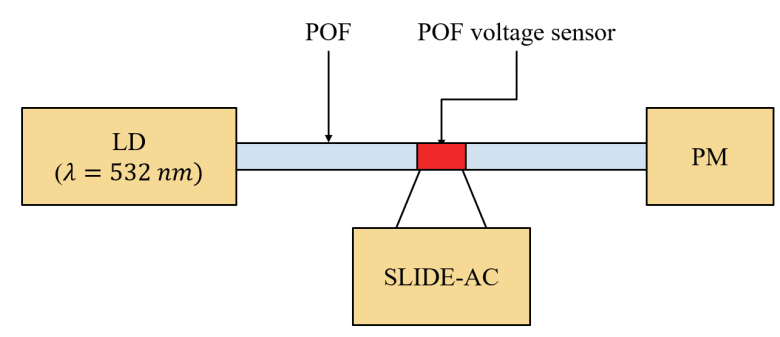

Fig. 3. (Color online) Experimental setup. 
532-nm continuous-wave laser diode (LD, Edmund Optics \#37-027), power meter (PM, Thorlabs PM100D), POF voltage sensor, and SLIDE-AC for changing the RMS amplitude of the AC voltage at $60 \mathrm{~Hz}$ applied to the POF voltage sensor. The light emitted from the LD was coupled into the POF and transmitted to the end of the POF. At the end of the POF, the optical power was read using the PM. When the light arrives at the PDLC film, it is partially transmitted and partially scattered. The light transmittance of the PDLC film depends on the amplitude of the AC voltage applied to the PDLC film. The optical power was measured by changing the RMS amplitude of the AC voltage at $60 \mathrm{~Hz}$, which is South Korea's standard electric power frequency, applied using the SLIDE-AC (Daelim Electric Co., 0.5K220). Then, the transmittance was calculated as $I(V) / I_{0}$, where $I(V)$ is the optical power at any applied voltage and $I_{0}$ is the optical power measured before the PDLC film was inserted into the rectangular hole in the POF.

Figure 4 shows a graph of the transmittance according to the RMS amplitude of the applied AC voltage $(60 \mathrm{~Hz})$. Figure 4(a) shows the transmittance as the RMS amplitude of the applied $\mathrm{AC}$ voltage is changed from 0 to $80 \mathrm{~V}$. Here, the transmittance is an exponential function
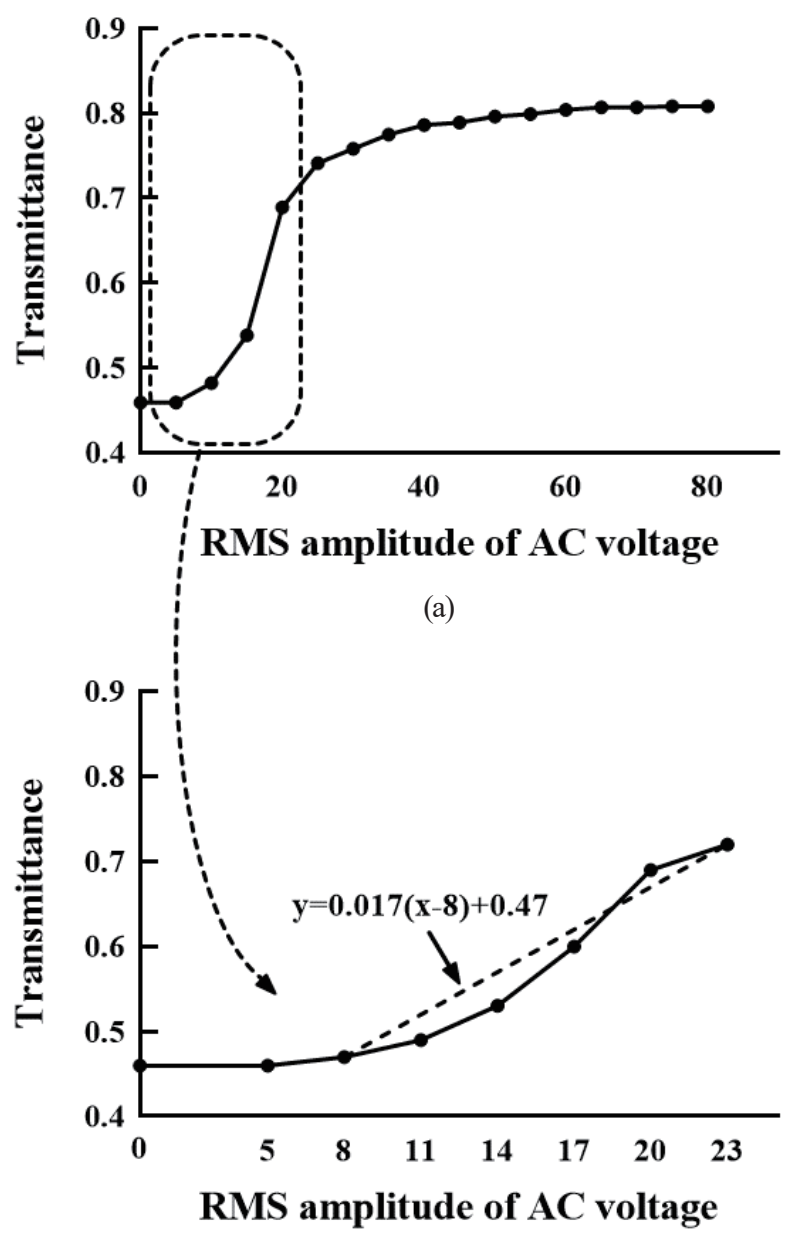

(b)

Fig. 4. Transmittance according to the RMS amplitude of AC voltage. (a) Transmittance as the RMS amplitude changes from 0 to $80 \mathrm{~V}$. (b) Transmittance as the RMS amplitude changes from 0 to $23 \mathrm{~V}$. 
of the RMS amplitude of the applied AC voltage like the Beer-Lambert Law formula. The transmittance is almost 0.46 from 0 to $5 \mathrm{~V}$. Then, the transmittance is proportional to the RMS amplitude of the applied voltage from 5 to $40 \mathrm{~V}$. Finally, the transmittance is saturated at approximately 0.8 over $40 \mathrm{~V}$. Figure 4(b) shows the transmittance as the RMS amplitude of the applied AC voltage is changed from 0 to $23 \mathrm{~V}$. Here, the transmittance increases almost linearly as the RMS amplitude of the applied voltage increases from 8 to $23 \mathrm{~V}$. In the approximate linear region, the sensitivity is approximately $0.017 \mathrm{~V}^{-1}$. In the linear region, the simple readout method of the RMS amplitude of the applied AC voltage is required. On the other hand, the complex signal processing readout algorithm is needed for more accurate measurement.

The results show that the POF voltage sensor with the rectangular hole filled with the PDLC film can be used to measure the RMS amplitude of AC voltage. Moreover, experimental results demonstrate that high-amplitude $\mathrm{AC}$ voltage can be measured if different PDLC films fabricated with different LCs, film thicknesses, and droplet concentrations are used.

\section{Conclusions}

The POF voltage sensor with a rectangular hole filled with a PDLC film was investigated. The dimensions of the rectangular hole of the POF and the dimensions of the PDLC film of were $2 \times 0.65 \mathrm{~mm}^{2}$ and $20 \times 0.6 \times 0.2 \mathrm{~mm}^{3}$, respectively. The POF voltage sensor was used to measure the RMS amplitude of $\mathrm{AC}$ voltage at $60 \mathrm{~Hz}$. The transmittance of the proposed POF voltage sensor was an exponential function of the RMS amplitude of the applied AC voltage like the Beer-Lambert Law formula. The sensitivity of the proposed POF voltage sensor was approximately $0.017 \mathrm{~V}^{-1}$ in the approximate linear region from 8 to $23 \mathrm{~V}$ where a simple readout method was required. The experimental results indicate that the proposed POF voltage sensor with the rectangular hole filled with the PDLC film can be used to measure the RMS amplitude of $\mathrm{AC}$ voltage $(60 \mathrm{~Hz})$. For more accurate measurement, the complex readout signal processing algorithm has to be developed. Moreover, research results show that high amplitude AC voltages can be measured using different PDLC films fabricated with different LCs, film thicknesses, and lower LC droplet concentrations.

\section{Acknowledgments}

This work was supported by the National Research Foundation (NRF) of Korea grant funded by the Korean government (MIST) No. 2018R1D1A1B07048066

\section{References}

1 J. Park: Opt. Eng. 50 (2011) 020501. https://doi.org/10.1117/1.3542040

2 A. Gowri and V. Sai: Sens. Actuators B 230 (2016) 536. https://doi.org/10.1016/j.snb.2016.02.074

3 D. Sartiano and S. Sales: Sensors 17 (2017) 2900. https://doi.org/10.3390/s17122900

4 C. Chu and Y. Lo: IEEE Photon. Technol. Lett. 20 (2008) 63. https://doi.org/10.1109/LPT.2007.912568

5 J. Zubia, L. Casado, G. Aldabaldetreku, A. Montero, E. Zubia, and G. Durana: Sensors 13 (2013) 13584. https://doi.org/10.3390/s131013584

6 M. Tabib, B. Sutapun, T. Srikhirin, J. Lando, and G. Adamovsky: Sens. Actuators, A 84 (2000) 134. https:// doi.org/10.1016/S0924-4247(99)00352-0 
7 J. N'cho and I. Fofana: Energies 13 (2020) 1789. https://doi.org/10.3390/en13071789

8 C. Li and T. Yoshino: J. Lightwave Technol. 20 (2002) 843. https://doi.org/10.1109/JLT.2002.1007938

9 V. Filippov, A. Starodumov, V. Monkovich, and Y. Barmenkov: IEEE Photon. Technol. Lett. 12 (2000) 870. https://doi.org/10.1109/JLT.2002.1007938

10 L.Martinez Leon, A. Diez, L. Cruz, and M. Andress: IEEE Photon. Technol. Lett. 13 (2001) 996. https://doi. org/10.1109/68.942672

11 S. Kim, J. Park, and W. Han: Microwave Opt. Technol. Lett. 51 (2009) 1689. https://doi.org/10.1002/mop.24434

12 J. Park: Jpn. J. Appl. Phys. 46 (2007) 3665. https://doi.org/10.1143/JJAP.46.3665

13 P. Niewczas, L. Dziuda, G. Fusiek, and J. McDonald: IEEE Trans. Instrum. Meas. 54 (2005) 1560. https://doi. org/10.1109/TIM.2004.851072

14 J. Lukens, N. Lagakos, V. Kaybulkin, C. Vizas, and D. King: IEEE Photon. Technol. Lett. 33 (2021) 1. https:// doi.org/10.1109/LPT2021.3052649

15 C. Lo and X. Cui: Sens. Actuators A 126 (2006) 62. https://doi.org/10.1016/j.sna.2005.09.013

16 E. Scherschener, C. Perciante, E. Dalchiele, E. Frins, M. Korn, and J. Ferrari: Appl. Opt. 45 (2006) 3482. https://doi.org/10.1364/AO.45.003482

17 J. Park and H. Seo: Sens. Mater. 32 (2002) 2117. https://doi.org/10.18494/SAM.2020.2773

18 Y. No and C. Jeon: Korean Chem. Eng. Res. 46 (2008) 938.

19 K. Hirabayashi, M. Wada, and C. Amano: IEEE Photon. Technol. Lett. 13 (2001) 487. https://doi. org $/ 10.1109 / 68.920760$

20 B. Lee, Y. Jeong, S. Baek, H. Kim, Y. Kim, Y. Lee, and S. Lee: Proc. 2002 15th Optical Fiber Sensors Conf. (IEEE, 2002) 403-406.

21 D. Ahn, Y. Park, J. Shin, J. Lee, and J. Park: Microwave Opt. Technol. Lett. 61 (2019) 120. https://doi. org/10.1002/mop.31524 